REGARDS

SUR L'ECONOMIE ALLEMAND

BULLETIN ECONOMIQUE DU CIRAC
Regards sur l'économie allemande

Bulletin économique du CIRAC

$84 \mid 2007$

Varia

\title{
Fiscalité des entreprises : une réforme à tiroirs
}

Isabelle Bourgeois

\section{CpenEdition}

Journals

Édition électronique

URL : http://journals.openedition.org/rea/567

DOI : $10.4000 /$ rea. 567

ISBN : 978-2-8218-0864-5

ISSN : 1965-0787

Éditeur

CIRAC

Édition imprimée

Date de publication : 1 décembre 2007

Pagination : 33-35

ISSN : 1156-8992

Référence électronique

Isabelle Bourgeois, "Fiscalité des entreprises : une réforme à tiroirs 》, Regards sur l'économie allemande [En ligne], 84 I décembre 2007, document 1, mis en ligne le 01 décembre 2009, consulté le 15 septembre 2020. URL : http://journals.openedition.org/rea/567

Ce document a été généré automatiquement le 15 septembre 2020.

(C) CIRAC 


\title{
Fiscalité des entreprises : une réforme à tiroirs
}

\author{
Isabelle Bourgeois
}

1 Le $1^{\text {er }}$ janvier 2008 entrera en vigueur le premier volet de la réforme de la fiscalité des entreprises. La mesure phare en est l'abaissement du taux de l'impôt sur les sociétés à $15 \%$ (il était de $25 \%$ ), ce qui, conjugué à diverses autres modifications de détail, se traduira par une baisse significative de l'impôt sur les bénéfices des sociétés de capitaux. Elles verront leur charge fiscale totale tomber de 38,65 \% (25\% d'IS, $17 \%$ de taxe professionnelle, le restant étant l'impôt de solidarité pour l'économie estallemande) à $29,83 \%$. L'Allemagne, qui se distinguait jusqu'ici par une fiscalité particulièrement élevée dans l'UE, rejoindra ainsi la moyenne européenne. Au $1^{\mathrm{er}}$ janvier 2009 entrera en vigueur l'autre mesure-phare de la Loi portant réforme de la fiscalité des entreprises (Unternehmenssteuerreformgesetz) et adoptée en seconde lecture par le Bundesrat le 6 juillet 2007 : un impôt libératoire au taux unique de $25 \%$ sur les revenus des capitaux privés (Abgeltungssteuer).

\section{Accroître l'attractivité du site et sécuriser les recettes fiscales}

2 Cette réforme qui met en œuvre la politique décidée par les partis au gouvernement fédéral lors de la conclusion du Contrat de coalition le 11 novembre 2005 (voir REA 74/05) vise un double bénéfice: accroître l'attractivité du site Allemagne pour les investisseurs étrangers (et allemands) dans un contexte européen caractérisé aujourd'hui par une plus grande mobilité du facteur capital, et dans le même temps "garantir sur le long terme les recettes fiscales", comme le présente le site du ministère fédéral des Finances (www.bundesfinanzministerium.de; rubrique «FAQ»). Car le problème de l'Allemagne, c'est un déséquilibre chronique entre recettes et dépenses.

3 Il ne s'agit donc nullement d'une baisse généralisée de la pression fiscale, qui serait incompatible avec l'objectif premier de la politique du gouvernement fédéral: la poursuite de la consolidation budgétaire. Une telle réduction ne pourra pas être 
envisagée avant 2010, explique le gouvernement. L'actuelle réforme prolonge celles engagées sous le gouvernement précédent, notamment dans le cadre de l'Agenda 2010, et dont les dispositions étaient entrées en vigueur progressivement depuis 2002 (voir REA 54/01 et 65/04). Les principales mesures en étaient la défiscalisation de la cession des plus-values réalisées par les sociétés de capitaux (2002), ainsi qu'un allègement de l'impôt sur le revenu, surtout des ménages (2004), suivi de la création d'un ISF (2007). Restait à revoir le régime s'appliquant aux entreprises.

\section{Le corollaire de la hausse de la TVA}

4 L'actuelle réforme s'inscrit aussi dans le contexte plus général d'un double rééquilibrage des prélèvements : d'une part entre impôts et cotisations, ces dernières représentant plus de $40 \%$ des prélèvements; d'autre part, entre fiscalité directe (en baisse continue depuis les années 1990) et indirecte. La hausse de 3 points de la TVA entrée en vigueur au $1^{\mathrm{er}}$ janvier 2007 (elle est destinée en partie à abaisser le taux des prélèvements sociaux pesant sur le travail) trouve ainsi aujourd'hui son corollaire dans une baisse de la fiscalité directe pesant sur les entreprises, les incitant à maintenir leurs activités sur le sol allemand, à y créer des emplois et à y payer leurs impôts.

\section{Un toilettage plus qu'une réforme de fond}

5 De l'aveu même du ministère fédéral de l'Economie (rapport mensuel de mai 2007), le choix effectué par le gouvernement de coalition est «pragmatique». Il ne s'est en effet pas inspiré des deux principaux modèles en débat depuis la fin 2003 et qui proposaient un changement de paradigme (voir REA 65/04). Le premier avait été développé par le Conseil des Sages dès son rapport annuel de 2003/04 et précisé dans celui de 2006/07; il préconise deux régimes distincts d'imposition pour les revenus du travail et du capital: « l'impôt dual » (Duale Einkommenssteuer). Le second est prôné par la Fondation Stiftung Marktwirtschaft (www.stiftung-marktwirtschaft.de) : un impôt synthétique, sorte de régime unique pour tous les revenus (travail, capital, etc.) et s'appliquant donc aux entreprises indépendamment de leur statut juridique (Allgemeine Unternehmenssteuer).

6 Le problème, dans les deux cas, résidait dans la nécessité de réformer en profondeur, voire de supprimer, la taxe professionnelle (Gewerbesteuer) - un impôt propre aux communes (voir REA 77/06). Or tout changement de régime de la fiscalité communale aurait nécessité au préalable une réflexion de fond sur l'architecture même du système fiscal allemand, autrement dit : une réforme globale du fédéralisme financier. Certes, la révision de ce dernier est actuellement en cours de négociation, mais l'objet n'en est que la répartition des compétences et ressources entre le Bund et les Länder.

7 Le gouvernement fédéral s'est donc contenté de toiletter le régime existant en retenant toutefois des éléments de ces deux approches, comme le révèle le nouveau caractère forfaitaire de l'impôt sur les revenus des capitaux privés, alors qu'est maintenue par ailleurs la progressivité de l'impôt sur le revenu du travail. Cela préfigure, à terme, l'option pour un régime "dual», comme l'estimait le Conseil des Sages dans son rapport 2006/07 au vu du projet de loi dont les éléments clefs ont été adoptés depuis. 


\section{Un coût limité à 5 milliards $€$}

8 Pour contrefinancer le manque à gagner induit par l'abaissement du taux de l'impôt sur les sociétés, l'assiette est élargie de diverses manières, et un certain nombre de niches fiscales (source d'évasion fiscale légale) sont supprimées. Ainsi, par exemple, une nouvelle "limite d'intérêts" (Zinsschranke) s'appliquera aux sociétés de capitaux internationalisées : comme les coûts d'intérêts déductibles seront calculés en fonction du bénéfice, cette mesure devrait les inciter à maintenir en Allemagne les activités bénéficiaires. Le coût de cette réforme ne devrait ainsi pas dépasser 5 milliards $€$, selon les estimations du gouvernement fédéral, partagées par le Conseil des Sages. Dans l'ensemble, "la réforme 2008 de la fiscalité des entreprises représente une amélioration par rapport au statu quo", étant donné que "les avantages l'emportent sur les inconvénients", conclut le Conseil des Sages dans son rapport 2007/08 rendu le 18 novembre.

\section{Une architecture problématique...}

9 Il n'en reste pas moins un certain nombre de points problématiques, soulignés par les différentes fédérations ou associations professionnelles selon la perspective qui leur est propre; elles restent donc nécessairement partielles en comparaison de l'évaluation des chances et risques effectuée par la Conseil des Sages qui replace les différentes mesures dans leur contexte macro-économique. Vu sous cet angle, le «tendon d'Achille » de la réforme provient d'une "articulation inadéquate " entre l'IS et l'impôt libératoire qui fausse les choix en matière de financement des entreprises puisqu'elle favorisera à partir de 2009 le recours au capital externe au lieu de promouvoir le financement en interne, qui était pourtant l'objectif déclaré du gouvernement fédéral.

\section{... et une fiscalité qui n'est pas neutre}

Cela risque non seulement de réduire l'investissement des entreprises non internationalisées, mais cela n'est pas neutre non plus en matière de choix de statut juridique (autre objectif initial) puisque les sociétés de capitaux (de la société par actions à la sarl) se trouvent favorisées par cette réforme. Cela est certes indispensable pour une économie ouverte, mais ces sociétés ne représentent que $15,4 \%$ des entreprises recensées en Allemagne, aux dires mêmes du ministère fédéral des Finances ; les sociétés de personnes sont majoritaires (82,6\%), et il s'agit pour beaucoup de PME artisanales.

\section{Les PME ne sont pas favorisées}

11 Pour les sociétés de personnes, le nouveau régime prévoit, sous conditions, un taux d'imposition plus avantageux pour les bénéfices thésaurisés qui se traduit, au total, par un rapprochement du taux d'imposition moyen entre les deux catégories de sociétés (29,77\% contre $29,83 \%)$. Mais en apparence seulement, explique le Conseil des Sages. En réalité, cette approche est problématique en droit du fait de la co-existence des deux régimes d'imposition applicables aux entreprises. La société de personnes se trouvant soumise à l'impôt sur le revenu (le même que celui des ménages), elle verra s'appliquer 
dès 2008 l'ISF allemand, dont le taux plafond est fixé à $45 \%$ pour les particuliers depuis janvier 2007. Au total, cela se traduira par un taux d'imposition effectif de $36,16 \%$ au lieu des $29,77 \%$ initialement escomptés. La loi ne tient donc pas ses promesses, qui étaient justement de veiller aussi à ne pas pénaliser les PME.

Le régime fiscal des entreprises allemandes diffère selon leur statut. Dans le cas des sociétés de personnes, le résultat de l'entreprise est attribué aux propriétaires qui doivent les imposer dans leur déclaration personnelle de revenus. L'entreprise n'est soumise qu'à la taxe professionnelle dont l'assiette n'est pas diminuée par d'éventuelles bonifications aux propriétaires. Cependant, la taxe professionnelle payée par l'entreprise est déductible du revenu imposable du ou des propriétaires. Ils ont la possibilité de compenser une perte provenant d'une participation dans une société de personnes avec leurs autres revenus.

Les sociétés de capitaux sont soumises à l'impôt sur les sociétés. Elles sont également soumises à la taxe professionnelle qui, contrairement aux sociétés de personnes, n'est pas imputée sur l'impôt sur le revenu des propriétaires de la société et représente ainsi une charge réelle supplémentaire pour l'entreprise. Cependant, la charge de la taxe professionnelle est souvent inférieure à celle des sociétés de personnes car des bonifications éventuelles versées aux propriétaires réduisent le bénéfice et ainsi l'assiette de la taxe professionnelle. Cependant, les propriétaires doivent déclarer les dividendes et intérêts reçus de l'entreprise et les imputer dans leur déclaration des revenus personnels. La société peut compenser des bénéfices par des pertes afin de réduire la charge fiscale, cette possibilité - contrairement aux sociétés de personnes - n'existe pas pour les propriétaires.

D'après: Gabel M., «Fiscalité : réforme structurelle et consolidation budgétaire », REA, n 77/2006.

\section{La réforme accroît la complexité du code fiscal}

Reste le principal grief : l'objectif d'une simplification du droit fiscal, objectif que se fixent tous les gouvernements allemands depuis de longues décennies, n'est pas atteint par cette réforme non plus. Au contraire, puisque cette réforme crée "un patchwork de variantes fiscales " pour les entreprises. La complexité des régimes possibles «devrait inciter à la recherche de montages visant à réduire la charge fiscale et dynamiser la branche du conseil fiscal », regrette le Conseil des Sages.

Les législateurs en semblent conscients, comme le révèle le fait que de nombreux correctifs à la loi de réforme ont été introduits dans la Loi fiscale annuelle 2008 (Jahressteuergesetz 2008) adoptée le 30 novembre en seconde lecture par le Bundesrat. La disposition clef en la matière est la nouvelle rédaction de l'art. 42 du Code des impôts qui vise à limiter le recours abusif aux possibilités de réductions fiscales ouvertes par la loi. Désormais, il revient au contribuable d'apporter la preuve que le régime d'imposition qu'il a choisi n'est pas guidé seulement par des motivations fiscales...

Cette réforme est loin d'être aboutie. Non seulement, elle n'apporte pas de simplification du droit, mais elle renforce une mauvaise allocation du capital en discriminant l'auto-financement de l'investissement par les sociétés œuvrant sur le territoire allemand. Elle ne bénéficie qu'aux sociétés déjà multi-positionnées hors des frontières. 


\section{Une articulation à trouver avec le droit des successions}

15 Par ailleurs, elle élude un autre aspect fondamental: le régime d'imposition des successions, stratégique pour l'avenir des sociétés familiales ( $95 \%$ des entreprises). Ce qui complique les décisions dans ce dossier en débat depuis de longues années (voir REA $65 / 04$ ), c'est notamment le fait que cet impôt revient en propre aux Länder. L'encours n'est certes pas énorme (de l'ordre de 4 milliards $€$ au total par an), mais est considéré comme un complément de ressources non négligeables par les Länder: il peut représenter jusqu'à $3 \%$ de leurs recettes fiscales, comme dans le cas de la Bavière (maximum). Or le patrimoine immobilier ou d'entreprise est moins imposé que les capitaux, ce qui avait amené en 2005 la Cour fédérale de juridiction financière à déclarer contraire à la Constitution le régime en vigueur et à renvoyer l'affaire sur le fond devant le Tribunal constitutionnel fédéral. Celui-ci avait publié le 31 janvier 2007 son arrêt du 7 novembre 2006 (1 BvL 10/02), confirmant l'absence d'équité du traitement fiscal des divers revenus et faisant injonction aux législateurs de réformer le code fiscal d'ici la fin 2008. Le 5 novembre 2007, la Commission ad hoc présidée par le ministre fédéral des Finances Peer Steinbrück (SPD) et le ministre-président de Hesse Roland Koch (CDU) a donc remis un projet de réforme, actuellement en discussion. Le pivot en est une nouvelle estimation des biens, désormais fondée sur leur valeur vénale; en contrepartie l'héritier bénéficie d'un abattement de $85 \%$ s'il reprend l'entreprise et en conserve le patrimoine pendant au moins 15 ans. Pour ne pas pénaliser les petites sociétés, le plancher d'imposition est fixé à $150000 €$.

\section{Et qui implique une réforme de l'impôt sur le revenu}

16 En théorie, selon le programme du gouvernement fédéral, et à condition que ce projet soit adopté par le parlement avant Noël, ces dispositions devraient entrer en vigueur le $1^{\mathrm{er}}$ avril. Rien n'est moins sûr, pour deux raisons au moins. L'approche des élections au Landtag de Hesse et de Basse-Saxe (27 janvier 2008) avive actuellement les différends entre SPD et CDU/CSU, d'autant que sur cet impôt se cristallisent traditionnellement tous les débats sur la 'justice' fiscale et la redistribution des revenus. Dans le même temps, la question de l'imposition des successions ne peut être traitée sérieusement hors de son contexte plus global: l'imposition des revenus. Autrement dit: les différents éléments (IS, IR et leurs déclinaisons) étant indissolublement liés, les réformes actuelles ne peuvent être considérées que comme un tout premier pas vers une réforme en profondeur de plus en plus urgente.

Car si l'abaissement du taux de l'IS à la moyenne européenne est un signal positif pour l'attractivité du site Allemagne, il s'accompagne de toute une série de mesures en contradiction avec le principe d'équité fiscale et risque, surtout, de se révéler contreproductif en comparaison des objectifs fixés, dont le changement de régime concernant la délocalisation de certaines fonctions (amendement apporté par la Loi Unternehmenssteuerreformgesetz au $\S 1,3$ de la Loi sur la fiscalité étrangère: Außensteuergesetz). Il vise à réduire l'évasion fiscale, en liaison avec 'l'évasion de savoirfaire'. Un décret doit venir préciser plusieurs points, dont la définition des « fonctions " tombant sous le coup de cette disposition, de même que son articulation avec les accords bilatéraux visant à éviter la double imposition... En réalité, explique une étude 
de l'institut DIW (Vierteljahrshefte 2/2007), "pour résoudre ces problèmes, il faudrait une coordination européenne et internationale accrue des réglementations nationales ». La réforme est donc loin d'être achevée...

INDEX

Mots-clés : entreprise, finances publiques, fiscalité, impôt, réforme 